Article

\title{
A Variable Acceptance Sampling Plan under Neutrosophic Statistical Interval Method
}

\author{
Muhammad Aslam (D) \\ Department of Statistics, Faculty of Science, King Abdulaziz University, Jeddah 21551, Saudi Arabia; \\ magmuhammad@kau.edu.sa or aslam_ravian@hotmail.com
}

Received: 3 January 2019; Accepted: 15 January 2019; Published: 19 January 2019

check for updates

\begin{abstract}
The acceptance sampling plan plays an important role in maintaining the high quality of a product. The variable control chart, using classical statistics, helps in making acceptance or rejection decisions about the submitted lot of the product. Furthermore, the sampling plan, using classical statistics, assumes the complete or determinate information available about a lot of product. However, in some situations, data may be ambiguous, vague, imprecise, and incomplete or indeterminate. In this case, the use of neutrosophic statistics can be applied to guide the experimenters. In this paper, we originally proposed a new variable sampling plan using the neutrosophic interval statistical method. The neutrosophic operating characteristic (NOC) is derived using the neutrosophic normal distribution. The optimization solution is also presented for the proposed plan under the neutrosophic interval method. The effectiveness of the proposed plan is compared with the plan under classical statistics. The tables are presented for practical use and a real example is given to explain the neutrosophic fuzzy variable sampling plan in the industry.
\end{abstract}

Keywords: optimization solution; sampling plan; producer's risk'; consumer's risk; sample size

\section{Introduction}

In this modern era, there is a strict competition between the companies to earn good reputation in the market. So, quality is considered as a benchmark for the well-reputed company. The good quality of the product means a good reputation of the company in the market. To maintain the high quality of the product, the inspection of the product from the raw material to the finished product should be done. Inspection of a finished lot of the products should be done before sending them to the market. Therefore, the inspection of the finished product is aimed at the high quality of the product. At the time of inspection, it may not possible to inspect 100\% of the items and the entire submitted lot of product. Therefore, inspection of a lot of product is done using the acceptance sampling plans. A well-designed sampling plan reduces the cost and time of the inspection. A sampling plan also pressures the producer to increase the quality of the product. As the decision about the submitted lot of product is taken based on the sample information, there is a chance of committing errors. The chance of rejecting a lot meets the given specification is called the producer's risk, and accepting a bad lot is known as the consumer's risk. Therefore, sampling plans are designed to give those parameters for the inspection of a lot of product, where these two risks are satisfied. More details about the sampling plans can be seen in $[1,2]$.

The variable sampling plan is applied when the data is continuous, such as the diameter of the ball bearing. Several variable acceptance sampling plans are available in the literature using classical statistics [1,3-5]. The variable sampling plans designed under classical statistics can only be applied when there is no certainty in the observations. According to [6] "observations include human judgments, and evaluations and decisions, a continuous random variable of a production process should include the variability caused by human subjectivity or measurement devices, or 
environmental conditions. These variability causes create vagueness in the measurement system". In this situation, the sampling plans designed use the fuzzy logic. Several authors presented excellent work to design the sampling plan under the fuzzy environment. Kanagawa and Ohta [7] designed the fuzzy attribute sampling plan. Jamkhaneh et al. [8] studied the rectifying fuzzy single sampling plan. Sadeghpour et al. [9] presented the plan with fuzzy parameters. Jamkhaneh et al. [10] discussed the effect of inspection errors on the single fuzzy plan. Tong and Wang [11] proposed the fuzzy sampling plan for the geospatial data. Turanoğlu et al. [12] presented the characteristic curve for the fuzzy plan. Uma and Ramya [13] presented a review of the fuzzy sampling plans. Kahraman et al. [14] worked on the single and double sampling using the fuzzy approach, and Afshari and Gildeh [15] designed a fuzzy multiple dependent state sampling plan.

According to [16], the neutrosophic logic is the generalization of classical fuzzy logic. The neutrosophic statistics developed by [17] is the generalization of the classical statistics. The neutrosophic statistics can be applied under the uncertainty environment. References $[18,19]$ introduced the neutrosophic interval method in rock measurement. Recently, references [20-25] introduced the neutrosophic statistics in the area of the acceptance sampling plan. Aslam [26] proposed a sampling plan for the exponential distribution using the neutrosophic statistics.

Although a rich variety of variable sampling plans under the fuzzy approach and classical statistics is available in the literature, according to the best of the author's knowledge, there is no work on the design of a variable sampling plan using the neutrosophic interval method. In this paper, we originally proposed a new variable sampling plan using the neutrosophic interval statistical method. The neutrosophic operating characteristic (NOC) is derived using the neutrosophic normal distribution. The optimization solution is also presented for the proposed plan under the neutrosophic statistical interval method. The effectiveness of the proposed plan is compared with the plan using classical statistics. The tables presented for practical use and a real example is given to explain the neutrosophic fuzzy variable sampling plan in the industry. A brief introduction to the neutrosophic approach is given in Section 2. The design of the proposed plan is given in Section 3. The advantages of the proposed plan are discussed in Section 4. An example is given in Section 5, and some concluding remarks are given in the last section.

\section{Neutrosophic Approach}

According to [16], the neutrosophic logic is an extension of fuzzy logic. The neutrosophic logic considers the measures of truth, false, and indeterminacy. The neutrosophic statistics using the neutrosophic logic is introduced by [17]. Classical statistics is the special case of the neutrosophic statistics. The latter one is applied when the sample is selected from the population having uncertain observations. According to [17] "neutrosophic statistics is an extension of the classical statistics. In the neutrosophic statistics, the data may be ambiguous, vague, imprecise, incomplete, even unknown. Instead of crisp numbers used in classical statistics, one uses sets in neutrosophic statistics". Suppose that $X_{N} \in\left\{X_{L}, X_{U}\right\}$ denotes the neutrosophic random variable, where $X_{L}$ and $X_{U}$ are lower and upper values of indeterminacy interval, respectively. Let $n_{N} \in\left\{n_{L}, n_{U}\right\}$ represent the neutrosophic sample size selected from the population having indeterminate observations. Let $\mu_{N} \in\left\{\mu_{L}, \mu_{U}\right\}$ and $\sigma_{N} \in\left\{\sigma_{L}, \sigma_{U}\right\}$ be the corresponding neutrosophic population mean and variance, respectively. Suppose that $s_{N} \in\left\{s_{L}, s_{U}\right\} \bar{X}_{N} \in\left\{\bar{X}_{L}, \bar{X}_{U}\right\}$ represent the neutrosophic sample mean and variance, respectively.

\section{Design of the Proposed Plan Neutrosophic Interval Method}

Based on the above information, in this section, we present the design of the proposed sampling under the neutrosophic environment. The operational procedure of the proposed plan is given as:

Step-1: Select a random sample of size $n_{L} \leq n_{N} \leq n_{U} ; n_{N} \in\left\{n_{L}, n_{U}\right\}$ from the lot of product. Compute the statistic $v_{N}=\frac{U-\bar{X}_{N}}{s_{N}}$, where $\bar{X}_{N} \in\left\{\bar{X}_{L}, \bar{X}_{U}\right\} ; \bar{X}_{L}=\sum_{i=1}^{n} x_{i}^{L} / n_{L}, \bar{X}_{U}=\sum_{i=1}^{n} x_{i}^{U} / n_{U}$, 
and $s_{N} \in\left\{s_{L}, s_{U}\right\}$, where $s_{L}=\sqrt{\sum_{i=1}^{n}\left(x_{i}^{L}-\bar{X}_{L}\right)^{2} / n_{L}}$ and $s_{U}=\sqrt{\sum_{i=1}^{n}\left(x_{i}^{U}-\bar{X}_{U}\right)^{2} / n_{U}} ; i=1,2,3$, $\ldots, n$.

Step-2: Accept the lot of product of $v \geq k_{N a} ; k_{N} \in\left\{k_{a L}, k_{a U}\right\}$ where $k_{N a}$ is the neutrosophic acceptance number.

The proposed plan is applied to test the hypothesis that the product is good versus the alternative hypothesis that the product is bad, on the basis of sample information. The null hypothesis is accepted if $v \geq k_{N}$, otherwise, the alternative hypothesis is accepted. The proposed plan has two parameters, $n_{N} \in\left\{n_{L}, n_{U}\right\}$ and $k_{N a} \in\left\{k_{a L}, k_{a U}\right\}$. The neutrosophic normal distribution, with mean $\mu_{N} \in\left\{\mu_{L}, \mu_{U}\right\}$ and standard deviation $\sigma_{N} \in\left\{\sigma_{L}, \sigma_{U}\right\}$, is defined by

$$
X_{N} \sim N_{N}\left(\mu_{N}, \sigma_{N}\right)=\frac{1}{\sigma_{N} \sqrt{2 \pi}} \exp \left(-\frac{\left(x-\mu_{N}\right)^{2}}{2 \sigma_{N}^{2}}\right)
$$

where $N_{N}\left(\mu_{N}, \sigma_{N}\right)$ denotes the neutrosophic normal distribution. The neutrosophic operating characteristic (NOC) of the proposed sampling plan is derived as follows:

Following [27], $\bar{X}_{N} \pm k_{N a} s_{N} ; \bar{X}_{N} \in\left\{\bar{X}_{L}, \bar{X}_{U}\right\}$ and $s_{N} \in\left\{s_{L}, s_{U}\right\}$ the approximate neutrosophic normal distribution with mean $\mu_{N} \pm c \sigma_{N} ; \mu_{N} \in\left\{\mu_{L}, \mu_{U}\right\} ; \sigma_{N} \in\left\{\sigma_{L}, \sigma_{U}\right\}$ and $\frac{\sigma_{N}^{2}}{n_{N}}+\frac{c^{2} \sigma_{N}^{2}}{2 n_{N}} ; \mu_{N} \in$ $\left\{\mu_{L}, \mu_{U}\right\} ; \sigma_{N} \in\left\{\sigma_{L}, \sigma_{U}\right\}$ and $n_{N} \in\left\{n_{L}, n_{U}\right\}$. The lot acceptance probability is given by

$$
L(p)=P\left(v_{N} \geq k_{N a}\right)=P\left\{\bar{X}_{N}+k_{N a} s_{N} \leq U\right\} ; \bar{X}_{N} \in\left\{\bar{X}_{L}, \bar{X}_{U}\right\}
$$

Therefore, the lot acceptance probability is given by

$$
L(p)=\Phi\left(\frac{U-\mu_{N}-k_{N a} s_{N}}{\left(\frac{\sigma_{N}}{\sqrt{n_{N}}}\right) \sqrt{1+\frac{k_{N a}^{2}}{2}}}\right) ; \bar{X}_{N} \in\left\{\bar{X}_{L}, \bar{X}_{U}\right\}, n_{N} \in\left\{n_{L}, n_{U}\right\}, k_{N a} \in\left\{k_{a L}, k_{a U}\right\} \text { and } s_{N} \in\left\{s_{L}, s_{U}\right\}
$$

Suppose $p_{U}$ is the probability that defective items beyond $U$, so $p_{U}=P\left(X_{N}>U \mid \mu_{N}\right)$; $X_{N} \in\left\{x_{i}^{L}, x_{i}^{U}\right\}$ and $\mu_{N} \in\left\{\mu_{L}, \mu_{U}\right\}$, where $Z_{N p_{U}}=\frac{U-\mu_{N}}{\sigma_{N}} ; \mu_{N} \in\left\{\mu_{L}, \mu_{U}\right\}$ and $Z_{N p_{U}}$ is the neutrosophic standard normal distribution. After some simplification, the NFOC is given by

$$
L_{N}(p)=\Phi\left(\left(Z_{N p_{U}}-k_{N a}\right) \sqrt{\frac{n_{N}}{1+\left(k_{N a}^{2} / 2\right)}}\right) ; k_{N a} \in\left\{k_{a L}, k_{a U}\right\} ; n_{N} \in\left\{n_{L}, n_{U}\right\}
$$

where $\alpha$ and $\beta$ are the producer's risk and consumer's risk, respectably. The plan parameters $k_{N a} \in\left\{k_{a L}, k_{a U}\right\} ; n_{N} \in\left\{n_{L}, n_{U}\right\}$ of the neutrosophic plan will be determined, such that the lot acceptance probability should be larger than $1-\alpha$ at acceptable quality level (AQL), and $p_{1}$ and bad lot acceptance probability should be smaller than $\beta$ at limiting quality level (LQL), say $p_{2}$. The neutrosophic plan parameters of the proposed sampling plans will be determined by the following non-linear optimization problem.

$$
\operatorname{minimize} n_{N} \in\left\{n_{L}, n_{U}\right\}
$$

subject to

$$
L_{N}\left(p_{1}\right)=\Phi\left(\left(Z_{N p_{U 1}}-k_{N a}\right) \sqrt{\frac{n_{N}}{1+\left(k_{N}^{2} / 2\right)}}\right) \geq 1-\alpha ; k_{N a} \in\left\{k_{a L}, k_{a U}\right\} ; n_{N} \in\left\{n_{L}, n_{U}\right\}
$$

and

$$
L_{N}\left(p_{2}\right)=\Phi\left(\left(Z_{N p_{U 2}}-k_{N a}\right) \sqrt{\frac{n_{N}}{1+\left(k_{N}^{2} a / 2\right)}}\right) \leq \beta ; k_{N} \in\left\{k_{a L}, k_{a U}\right\} ; n_{N} \in\left\{n_{L}, n_{U}\right\}
$$


The neutrosophic plan parameters such as $n_{N} \in\left\{n_{L}, n_{U}\right\}, k_{N a} \in\left\{k_{a L}, k_{a U}\right\}, L_{N}\left(p_{1}\right)$, and $L_{N}\left(p_{2}\right)$ for various values of AQL and LQL are placed in Table 1.

Table 1. The neutrosophic plan parameter when $\alpha=0.05, \beta=0.05$.

\begin{tabular}{cccccc}
\hline$p_{1}$ & $p_{2}$ & $n_{N}$ & $k_{N a}$ & $L_{N}\left(p_{1}\right)$ & $L_{N}\left(p_{2}\right)$ \\
\hline 0.001 & 0.002 & $\{388,569\}$ & $\{1.062,1.068\}$ & $\{0.0151,0.065\}$ & $\{0.9501,0.9502\}$ \\
& 0.003 & $\{213,268\}$ & $\{1.05,1.055\}$ & $\{0.000,0.000\}$ & $\{0.9501,0.9502\}$ \\
& 0.004 & $\{180,213\}$ & $\{1.046,1.05\}$ & $\{0.000,0.000\}$ & $\{0.9500,0.9502\}$ \\
& 0.006 & $\{139,150\}$ & $\{1.039,1.041\}$ & $\{0.000,0.000\}$ & $\{0.9504,0.9508\}$ \\
& 0.008 & $\{103,130\}$ & $\{1.03,1.037\}$ & $\{0.000,0.000\}$ & $\{0.9500,0.9506\}$ \\
& 0.010 & $\{78,117\}$ & $\{1.02,1.034\}$ & $\{0.000,0.026\}$ & $\{0.9501,0.9506\}$ \\
& 0.015 & $\{61,100\}$ & $\{1.01,1.029\}$ & $\{0.000,0.007\}$ & $\{0.9500,0.9512\}$ \\
0.0025 & 0.020 & $\{50,80\}$ & $\{0.99,1.02\}$ & $\{0.000,0.010\}$ & $\{0.9527,0.6983\}$ \\
& 0.030 & $\{143,233\}$ & $\{0.696,0.706\}$ & $\{0.0173,0.0847\}$ & $\{0.9501,0.9504\}$ \\
0.005 & 0.050 & $\{138,213\}$ & $\{0.695,0.705\}$ & $\{0.0214,0.0937\}$ & $\{0.9504,0.9506\}$ \\
& 0.050 & $\{77,83\}$ & $\{0.708,0.711\}$ & $\{0.9999,1.000\}$ & $\{0.0791,0.0990\}$ \\
0.01 & 0.100 & $\{15,22\}$ & $\{0.77,0.795\}$ & $\{0.0012,0.0118\}$ & $\{0.9545,0.9577\}$ \\
& 0.020 & $\{184,210\}$ & $\{0.812,0.814\}$ & $\{0.0731,0.0985\}$ & $\{0.9500,0.9536\}$ \\
0.03 & 0.030 & $\{95,102\}$ & $\{0.794,0.796\}$ & $\{0.0275,0.0358\}$ & $\{0.9502,0.9508\}$ \\
& 0.060 & $\{127,149\}$ & $\{0.669,0.673\}$ & $\{0.0638,0.0992\}$ & $\{0.9516,0.9520\}$ \\
0.05 & 0.090 & $\{112,121\}$ & $\{0.666,0.668\}$ & $\{0.0010,0.0018\}$ & $\{0.9502,0.9507\}$ \\
& 0.100 & $\{125,132\}$ & $\{0.60,0.614\}$ & $\{0.0029,0.0551\}$ & $\{0.9501,0.9503\}$ \\
& 0.150 & $\{38,40\}$ & $\{0.557,0.559\}$ & $\{0.0812,0.0921\}$ & $\{0.9512,0.9519\}$ \\
\hline
\end{tabular}

From Table 1, we note following trends in neutrosophic plan parameters

1. For the fixed values AQL, $n_{N} \in\left\{n_{L}, n_{U}\right\}$ decreases as LQL increases.

2. For the fixed values $\mathrm{AQL}, k_{\mathrm{Na}} \in\left\{k_{a L}, k_{a u}\right\}$ decreases as LQL increases.

\section{Comparative Study}

Now we compare the proposed plan with the sampling plan under classical statistics in [19], a method which provides the range of the parameters under the uncertainty is called the most effective and adequate method. We preened the values of both sampling plans for some combinations of AQL and LQL in Table 2. From Table 2, it can be noted that the plan under classical statistics provides the determined value, while the proposed plan provides the plan parameter in the indeterminacy interval. For example, when $\mathrm{AQL}=0.001$ and $\mathrm{LQL}=0.002$, the proposed plan has indeterminacy interval $n_{N} \in\{388,569\}$, while the plan under classical statistics has a determined value $n=388$. Under the uncertainty, when $\mathrm{AQL}=0.001$ and $\mathrm{LQL}=0.002$, the suitable sample size should be selected between 388 and 569. Therefore, the sampling plan under the neutrosophic interval method has the advantage over the plan under classical statistics under the uncertainty environment. The proposed plan is more effective, informative, flexible, and adequate to be applied in uncertainty than the plan based on classical statistics.

Table 2. The comparison of neutrosophic plan with plan under classical Statistics, when $\alpha=0.05, \beta=0.05$.

\begin{tabular}{cccc}
\hline \multirow{2}{*}{$p_{\mathbf{1}}$} & $p_{\mathbf{2}}$ & Proposed Plan & Existing Plan \\
\cline { 3 - 4 } & & $n_{N}$ & $\boldsymbol{n}$ \\
\hline 0.001 & 0.002 & $\{388,569\}$ & 388 \\
0.001 & 0.010 & $\{78,117\}$ & 78 \\
0.001 & 0.020 & $\{50,80\}$ & 50 \\
0.005 & 0.050 & $\{77,83\}$ & 77 \\
0.005 & 0.100 & $\{15,22\}$ & 15 \\
0.01 & 0.020 & $\{184,210\}$ & 184 \\
0.01 & 0.030 & $\{95,102\}$ & 95 \\
0.05 & 0.100 & $\{125,132\}$ & 125 \\
\hline
\end{tabular}




\section{Application of the Proposed Plan}

In this section, we present the application of the proposed neutrosophic plan using color STN display data collected from the industry of LCD. According to [28], "Color STN displays are created by adding color filters to traditional monochrome. In color STN displays, each pixel is divided into R, $\mathrm{G}$, and B sub-pixels. In this study, the membrane thickness of each pixel is the quality characteristic". The data for this variable of study is obtained from the measurement process. Senturk and Erginel [6] pointed out that the observations obtained from the measurement devices have variability. The present state of this variation makes some observations imprecise. During the sample study, we found that some observations about each pixel are determinate or clear, and some are indeterminate or unclear. For this experiment, the experimenter did not determinate the sample size that should be selected for the inspection of this LCD product. Suppose we fixed $\alpha=0.05, \beta=0.05, \mathrm{AQL}=0.001$, and LQL $=0.020$. From Table 1, it can be noted that the optimal sample size $n_{N}$ for this case should be $n_{N} \in\{50,80\}$. Therefore, we need to collect the data for a sample size having between 50 and 80 . Let $n_{N}=55$ once $n_{N} \in\{50,80\}$. The data of 55 observations, including determinate and indeterminate observations about each pixel, is reported in Table 3.

Table 3. Data on color Super Twisted Nematic (STN) displays.

$[11,816.7,11,816.7][11,710.1,11,710.1][11,722.6,11,823.5][11,744.1,11,744.1][11,681.1,11,681.1][11,728.4,11,728.4]$

$[11,712.6,11,712.6][11,775.2,11,775.2][11,743.3,11,743.3][11,786.1,11,786.1][11,760.6,11,760.6][11,723.6,11,723.6]$

$[11,721.7,11,721.7][11,698,11,698] \quad[11,695.9,11,695.9][11,726.4,11,726.4][111,797.2,11,797.2][11,773.1,11,773.1]$

$[11,769.1,11,769.1][11,800.8,11,800.8][11,780.7,11,780.7][11,670.9,11,675.9][111,692.3,11,692.3][11,666.2,11,666.2]$

$[11,755.2,11,762.5][11,712.7,11,712.7][11,775.5,11,775.5][11,731.2,11,731.2][111,625.6,11,625.6][11,757.5,11,757.5]$

$[11,674.7,11,674.7][11,729.2,11,729.2][11,681.3,11,681.3][11,636.4,11,636.4][11,682.1,11,690.7][11,667.9,11,667.9]$

$[11,722.9,11,722.9][11,655.3,11,655.3][11,700.2,11,700.2][11,754.2,11,754.2][111,769.9,11,769.9][11,705.9,11,705.9]$

$[11,589.8,11,589.8][11,738.4,11,745.6][11,745.4,11,745.4][11,727.7,11,727.7][11,664.3,11,664.3][11,647.2,11,647.2]$

$[11,755,11,755] \quad[11,671.8,11,671.8][11,705.8,11,705.8][11,664.2,11,664.2][11,677.0,11,695.2][111,680.5,11,687.4]$ $[11,633.6,11,633.6]$

As the data given in Table 3 is neutrosophic, therefore, the sampling plan under classical statistics cannot be applied for the inspection of this product. The proposed sampling plan for the neutrosophic data is explained as follows.

By following [17], the sample mean $\bar{X}_{N} \in\left\{\bar{X}_{L}, \bar{X}_{U}\right\}$ and $s_{N} \in\left\{s_{L}, s_{U}\right\}$ for this data are calculated as follows

$$
\bar{X}_{N}=\frac{[11,816.7,11,816.7]+[11,710.1,11,710.1]+[11,722.6,11,722.6]+\ldots+[11,633.6,11,633.6],[11,816.7,11,816.7]+\ldots}{+[11,687.4,11687.4]+[11,633.6,11,633.6]}
$$

Or

$$
\bar{X}_{N} \in\{11,715.2,11,719.6\} \text { and } s_{N}=\{49.21,49.70\}
$$

Suppose that $U=12,500$ for the submitted LCD product. The proposed sampling plan implemented as follows.

Step-1: select a random sample of size $n_{N}=\left\{n_{L}, n_{U}\right\}$ from a lot of product. Compute the statistic $v_{N}=\frac{U-\bar{X}_{N}}{s_{N}}=\frac{[12,500,12,500]-[11,715.2,11,719.6]}{[49.21,49.70]}$, so $v_{N} \in[15.70,15.94]$.

Step-2: Accept a lot of the product of $v_{N} \geq k_{N} ; k_{N a} \in\left\{k_{a L}, k_{a u}\right\}$. From Table 1, we have $k_{N a} \in\{0.99$, $1.02\}$. So, $v_{N} \geq k_{N a}$, the lot of product, should be accepted to send to the market.

From this real example, it is concluded that the proposed sampling is quite reasonable and adequate to apply when observations are imprecise. 


\section{Concluding Remarks}

A new variable neutrosophic variable sampling plan is proposed in this paper. The proposed plan is the extension of the variable sampling plan based on classical statistics. The proposed plan can be applied in those situations where data is incomplete or indeterminate came from the complex process. The non-linear optimization problem was developed under the neutrosophic approach, and some results are presented for the practical use of the proposed plan. A real example shows the application of the proposed plan in the industry. From the comparison study, it was concluded that the proposed plan under the neutrosophic interval method is an adequate, flexible, effective, and reasonable method in the uncertainty environment. The proposed plan has the limitation that it can be applied only when the data follows the neutrosophic normal distribution. The proposed plan, using some other sampling scheme, will be considered for future research. The proposed plan for some non-normal distribution can be extended as future research.

Funding: This work was supported by the Deanship of Scientific Research (DSR), King Abdulaziz University, Jeddah. The author, Muhammad Aslam, therefore, acknowledges with thanks DSR technical support.

Acknowledgments: The author is deeply thankful to the editor and reviewers for their valuable suggestions to improve the quality of this manuscript.

Conflicts of Interest: The author declares no conflict of interest regarding this paper.

\section{References}

1. Montgomery, D.C. Introduction to Statistical Quality Control; John Wiley \& Sons: Hoboken, NJ, USA, 2007.

2. Aslam, M.; Wu, C.-W.; Azam, M.; Jun, C.-H. Variable sampling inspection for resubmitted lots based on process capability index Cpk for normally distributed items. Appl. Math. Model. 2013, 37, 667-675. [CrossRef]

3. Balamurali, S.; Jun, C.-H. Repetitive group sampling procedure for variables inspection. J. Appl. Stat. 2006, 33, 327-338. [CrossRef]

4. Jun, C.-H.; Balamurali, S.; Lee, S.-H. Variables sampling plans for Weibull distributed lifetimes under sudden death testing. IEEE Trans. Reliab. 2006, 55, 53-58. [CrossRef]

5. Wu, C.-W.; Aslam, M.; Jun, C.-H. Developing a variables two-plan sampling system for product acceptance determination. Commun. Stat. Theory Methods 2017, 46, 706-720. [CrossRef]

6. Senturk, S.; Erginel, N. Development of fuzzy $\mathrm{X}^{-} \sim-\mathrm{R} \sim$ and $\mathrm{X}^{-} \sim-\mathrm{S} \sim$ control charts using $\alpha$-cuts. Inf. Sci. 2009, 179, 1542-1551. [CrossRef]

7. Kanagawa, A.; Ohta, H. A design for single sampling attribute plan based on fuzzy sets theory. Fuzzy Sets Syst. 1990, 37, 173-181. [CrossRef]

8. Jamkhaneh, E.B.; Sadeghpour-Gildeh, B.; Yari, G. Important criteria of rectifying inspection for single sampling plan with fuzzy parameter. Int. J. Contemp. Math. Sci. 2009, 4, 1791-1801.

9. Sadeghpour Gildeh, B.; Baloui Jamkhaneh, E.; Yari, G. Acceptance single sampling plan with fuzzy parameter. Iran. J. Fuzzy Syst. 2011, 8, 47-55.

10. Jamkhaneh, E.B.; Sadeghpour-Gildeh, B.; Yari, G. Inspection error and its effects on single sampling plans with fuzzy parameters. Struct. Multidiscip. Optim. 2011, 43, 555-560. [CrossRef]

11. Tong, X.; Wang, Z. Fuzzy acceptance sampling plans for inspection of geospatial data with ambiguity in quality characteristics. Comput. Geosci. 2012, 48, 256-266. [CrossRef]

12. Turanoğlu, E.; Kaya, İ.; Kahraman, C. Fuzzy acceptance sampling and characteristic curves. Int. J. Comput. Intell. Syst. 2012, 5, 13-29. [CrossRef]

13. Uma, G.; Ramya, K. Impact of Fuzzy Logic on Acceptance Sampling Plans-A Review. Autom. Auton. Syst. 2015, 7, 181-185.

14. Kahraman, C.; Bekar, E.T.; Senvar, O. A Fuzzy Design of Single and Double Acceptance Sampling Plans. In Intelligent Decision Making in Quality Management; Springer: Berlin/Heidelberg, Germany, 2016; pp. 179-211.

15. Afshari, R.; Gildeh, B.S. Construction of fuzzy multiple deferred state sampling plan. In Proceedings of the Fuzzy Systems Association and 9th International Conference on Soft Computing and Intelligent Systems (IFSA-SCIS), 2017 Joint 17th World Congress of International, Otsu, Japan, 27-30 June 2017; pp. 1-7. 
16. Smarandache, F. Neutrosophic Logic-A Generalization of the Intuitionistic Fuzzy Logic. In Multispace $\mathcal{E}$ Multistructure Neutrosophic Transdisciplinarity (100 Collected Papers of Science); Infinite Study: Conshohocken, PA, USA, 2010; Volume 4, p. 396.

17. Smarandache, F. Introduction to Neutrosophic Statistics; Infinite Study: Conshohocken, PA, USA, 2014.

18. Chen, J.; Ye, J.; Du, S. Scale effect and anisotropy analyzed for neutrosophic numbers of rock joint roughness coefficient based on neutrosophic statistics. Symmetry 2017, 9, 208. [CrossRef]

19. Chen, J.; Ye, J.; Du, S.; Yong, R. Expressions of rock joint roughness coefficient using neutrosophic interval statistical numbers. Symmetry 2017, 9, 123. [CrossRef]

20. Aslam, M. A New Sampling Plan Using Neutrosophic Process Loss Consideration. Symmetry 2018, 10, 132. [CrossRef]

21. Aslam, M.; Arif, O. Testing of Grouped Product for the Weibull Distribution Using Neutrosophic Statistics. Symmetry 2018, 10, 403. [CrossRef]

22. Aslam, M.; Raza, M.A. Design of New Sampling Plans for Multiple Manufacturing Lines Under Uncertainty. Int. J. Fuzzy Syst. 2018, 1-15. [CrossRef]

23. Aslam, M. A new attribute sampling plan using neutrosophic statistical interval method. Complex. Intell. Syst. 2019, 1-6. [CrossRef]

24. Aslam, M. A New Failure-Censored Reliability Test Using Neutrosophic Statistical Interval Method. Int. J. Fuzzy Syst. 2019, 1-7. [CrossRef]

25. Aslam, M. Product Acceptance Determination with Measurement Error Using the Neutrosophic Statistics. Adv. Fuzzy Syst. 2019, 2019, 8953051. [CrossRef]

26. Aslam, M. Design of Sampling Plan for Exponential Distribution under Neutrosophic Statistical Interval Method. IEEE Access 2018, 6, 64153-64158. [CrossRef]

27. Duncan, A.J. Quality Control and Industrial Statistics; R. D. Irwin: Homewood, IL, USA, 1974.

28. Aslam, M.; Yen, C.-H.; Chang, C.-H.; Jun, C.-H.; Ahmad, M.; Rasool, M. Two-stage variables acceptance sampling plans using process loss functions. Commun. Stat. Theory Methods 2012, 41, 3633-3647. [CrossRef]

(C) 2019 by the author. Licensee MDPI, Basel, Switzerland. This article is an open access article distributed under the terms and conditions of the Creative Commons Attribution (CC BY) license (http:/ / creativecommons.org/licenses/by/4.0/). 